Research Article

\title{
Approximate Controllability of a Reaction-Diffusion System with a Cross-Diffusion Matrix and Fractional Derivatives on Bounded Domains
}

\author{
Salah Badraoui \\ Laboratoire LAIG, Université du 08 Mai 1945, BP. 401, Guelma 24000, Algeria \\ Correspondence should be addressed to Salah Badraoui, sabadraoui@hotmail.com \\ Received 11 July 2009; Accepted 5 January 2010 \\ Academic Editor: Ugur Abdulla \\ Copyright (C) 2010 Salah Badraoui. This is an open access article distributed under the Creative \\ Commons Attribution License, which permits unrestricted use, distribution, and reproduction in \\ any medium, provided the original work is properly cited. \\ We study the following reaction-diffusion system with a cross-diffusion matrix and fractional \\ derivatives $u_{t}=a_{1} \Delta u+a_{2} \Delta v-c_{1}(-\Delta)^{\alpha_{1}} u-c_{2}(-\Delta)^{\alpha_{2}} v+1_{\omega} f_{1}(x, t)$ in $\left.\Omega \times\right] 0, t^{*}\left[, v_{t}=b_{1} \Delta u+b_{2} \Delta v-\right.$ \\ $d_{1}(-\Delta)^{\beta_{1}} u-d_{2}(-\Delta)^{\beta_{2}} v+1_{\omega} f_{2}(x, t)$ in $\left.\Omega \times\right] 0, t^{*}[, u=v=0$ on $\partial \Omega \times] 0, t^{*}\left[, u(x, 0)=u_{0}(x), v(x, 0)=\right.$ \\ $v_{0}(x)$ in $x \in \Omega$, where $\Omega \subset \mathbb{R}^{N}(N \geq 1)$ is a smooth bounded domain, $u_{0}, v_{0} \in L^{2}(\Omega)$, the diffusion \\ matrix $M=\left(\begin{array}{ll}a_{1} & a_{2} \\ b_{1} & b_{2}\end{array}\right)$ has semisimple and positive eigenvalues $0<\rho_{1} \leq \rho_{2}, 0<\alpha_{1}, \alpha_{2}, \beta_{1}, \beta_{2}<1$, \\ $\omega \subset \Omega$ is an open nonempty set, and $1_{\omega}$ is the characteristic function of $\omega$. Specifically, we prove \\ that under some conditions over the coefficients $a_{i}, b_{i}, c_{i}, d_{i}(i=1,2)$, the semigroup generated by \\ the linear operator of the system is exponentially stable, and under other conditions we prove that \\ for all $t^{*}>0$ the system is approximately controllable on $\left[0, t^{*}\right]$.
}

\section{Introduction}

In this paper we prove controllability for the following reaction-diffusion system with cross diffusion matrix:

$$
\begin{gathered}
\left.u_{t}=a_{1} \Delta u+a_{2} \Delta v-c_{1}(-\Delta)^{\alpha_{1}} u-c_{2}(-\Delta)^{\alpha_{2}} v+1_{\omega} f_{1}(x, t) \text { in } \Omega \times\right] 0, t^{*}[, \\
\left.v_{t}=b_{1} \Delta u+b_{2} \Delta v-d_{1}(-\Delta)^{\beta_{1}} u-d_{2}(-\Delta)^{\beta_{2}} v+1_{\omega} f_{2}(x, t) \text { in } \Omega \times\right] 0, t^{*}[, \\
u=v=0 \quad \text { on } \partial \Omega \times] 0, t^{*}[, \\
u(x, 0)=u_{0}(x), \quad v(x, 0)=v_{0}(x) \quad \text { in } x \in \Omega,
\end{gathered}
$$

where $\omega$ is an open nonempty set of $\Omega$ and $1_{\omega}$ is the characteristic function of $\omega$. 
We assume the following assumptions.

(H1) $\Omega$ is a smooth bounded domain in $\mathbb{R}^{N}(N \geq 1)$.

(H2) The diffusion matrix $M=\left(\begin{array}{ll}a_{1} & a_{2} \\ b_{1} & b_{2}\end{array}\right)$ has semisimple and positive eigenvalues $0<\rho_{1} \leq$ $\rho_{2}$.

(H3) $c_{j}, d_{j}(j=1,2)$ are real constants, $\alpha_{j}, \beta_{j}(j=1,2)$ are real constants belonging to the interval $] 0,1[$.

(H4) $u_{0}, v_{0} \in L^{2}(\Omega)$.

(H5) The distributed controls $f_{1}, f_{2} \in L^{2}\left(\left[0, t^{*}\right] ; L^{2}(\Omega)\right)$.

Specifically, we prove the following statements.

(i) If $c_{2}=d_{1}=0$ and $\min \left\{c_{1}+\lambda_{1}^{1-\alpha_{1}} \rho_{1}, d_{2}+\lambda_{1}^{1-\beta_{2}} \rho_{1}\right\}>0$, where $\lambda_{1}$ is the first eigenvalue of $-\Delta$ with Dirichlet condition, or if $c_{2} \neq 0, d_{1} \neq 0, c_{1} \geq 0$, and $d_{2} \geq 0$; then, under the hypotheses $(\mathrm{H} 1)-(\mathrm{H} 3)$, the semigroup generated by the linear operator of the system is exponentially stable.

(ii) If $c_{2}=d_{1}=0$ and under the hypotheses (H1)-(H5), then, for all $t^{*}>0$ and all open nonempty subset $\omega$ of $\Omega$ the system is approximately controllable on $\left[0, t^{*}\right]$.

This paper has been motivated by the work done in [1] and the work done by H. Larez and H. Leiva in [2]. In the work [1], the auther studies the asymptotic behavior of the solution of the system

$$
\begin{array}{ll}
u_{t}=a \frac{\partial^{2} u}{\partial x^{2}}+\beta \frac{\partial u}{\partial x}+b \frac{\partial^{2} v}{\partial x^{2}}+f(t, u, v), & x \in \mathbb{R}, t>0 \\
v_{t}=c \frac{\partial^{2} u}{\partial x^{2}}+d \frac{\partial^{2} v}{\partial x^{2}}+\beta \frac{\partial v}{\partial x}+g(t, u, v), & x \in \mathbb{R}, t>0
\end{array}
$$

supplemeted with the initial conditions

$$
u(x, 0)=u_{0}(x), \quad v(x, 0)=v_{0}(x), \quad x \in \mathbb{R} .
$$

The author proved that in the Banach space $X \times X$ where $X=C_{\mathrm{ub}}(\mathbb{R})$ is the space of bounded uniformly continuous real valued functions on $\mathbb{R}$, if $f$ and $g$ are locally Lipshitz and under some conditions over the coefficients $a, b, c, d, \beta$, and if $u_{0}, v_{0} \in C_{+}=\left\{u \in C_{\mathrm{ub}}(\mathbb{R})\right.$ : $\lim _{x \rightarrow+\infty} u(x)$ exist $\}$, then $u(t), v(t) \in C_{+}$for all $t<t_{\text {max }}$. Moreover, $U(t)=\lim _{x \rightarrow+\infty} u(x)$ and $V(t)=\lim _{x \rightarrow+\infty} v(x)$ satisfy the system of ordinary differential equations

$$
\begin{aligned}
& U^{\prime}(t)=f(t, U(t), V(t), \\
& V^{\prime}(t)=g(t, U(t), V(t)
\end{aligned}
$$


with the initial data

$$
U(0)=\lim _{x \rightarrow+\infty} u_{0}(x), \quad V(0)=\lim _{x \rightarrow+\infty} v_{0}(x)
$$

The same result holds for $C_{-}=\left\{u \in C_{\mathrm{ub}}(\mathbb{R}): \lim _{x \rightarrow-\infty} u(x)\right.$ exist $\}$.

In the work done in [2], the authers studied the system (1.1) with $c_{2}=d_{1}=0, c_{1}=d_{2}$, and $\alpha_{1}=\beta_{2}=1 / 2$. They proved that if the diffusion matrix $\left(\begin{array}{ll}a & b \\ c & d\end{array}\right)$ has semi-simple and positive eigenvalues $0<\rho_{1} \leq \rho_{2}, f_{1}, f_{2} \in L^{2}\left(\left[0, \tau\left[; L^{2}(\Omega)\right)\right.\right.$, then if $\lambda_{1}^{1 / 2} \rho_{1}+\beta>0\left(\lambda_{1}\right.$ is the first eigenvalue of $-\Delta)$, the system is approximately controllable on $[0, \tau]$ for all open nonempty subset $\omega$ of $\Omega$.

\section{Notations and Preliminaries}

In the following we denote by

$\mathcal{M}_{2}(\mathbb{R})$ the set of $2 \times 2$ matrices with entries from $\mathbb{R}$,

$L^{2}(\Omega)$ the set of all measurable functions $u: \Omega \rightarrow \mathbb{R}$ such that $\int_{\Omega}|u|^{2} d x<\infty$,

$H^{1}(\Omega)$ the set of all the functions $u \in L^{2}(\Omega)$ that have generalized derivatives $\partial u / \partial x_{j} \in L^{2}(\Omega)$ for all $j=1, \ldots, N$,

$H_{0}^{1}(\Omega)$ the closure of the set $C_{0}^{\infty}(\Omega)$ in the Hilbert space $H^{1}(\Omega)$,

$H^{2}(\Omega)$ the set of all the functions $u \in L^{2}(\Omega)$ that have generalized derivatives $\partial u / \partial x_{j}, \partial^{2} u / \partial x_{j} \partial x_{k} \in L^{2}(\Omega)$ for all $j, k=1, \ldots, N$.

We will use the following results.

Theorem 2.1 (cf. [3]). Let us consider the following classical boundary-eigenvalue problem for the laplacien:

$$
\begin{gathered}
-\Delta u=\lambda u, \quad \text { on } \Omega, \\
u=0, \quad \text { on } \partial \Omega,
\end{gathered}
$$

where $\Omega$ is a nonempty bounded open set in $\mathbb{R}^{N}$ and $D(-\Delta)=H^{2}(\Omega) \cap H_{0}^{1}(\Omega)$.

This problem has a countable system of eigenvalues $0<c \leq \lambda_{1}<\lambda_{2}<\cdots<\lambda_{j}<\cdots$ and $\lambda_{j} \rightarrow+\infty$ as $j \rightarrow \infty$.

(i) All the eigenvalues $\lambda_{j}$ have finite multiplicity $m_{j}$ equal to the dimension of the corresponding eigenspace $S_{j}$.

(ii) Let $\left\{\varphi_{j k}\right\}_{k=1}^{m_{j}}$ be a basis of the $S_{j}$ for every $j$, then the eigenvectors $\left\{\varphi_{j k}\right\}_{k=1, j=1}^{m_{j, \infty}}$ form a complete orthonormal system in the space $L^{2}(\Omega)$. Hence for all $u \in L^{2}(\Omega)$ we have $u=\sum_{j=1}^{\infty} \sum_{k=1}^{m_{j}}\left\langle u, \varphi_{j k}\right\rangle \varphi_{j k}$. If we put $E_{j} u=\sum_{k=1}^{m_{j}}\left\langle u, \varphi_{j k}\right\rangle \varphi_{j k}$ then we get $u=$ $\sum_{j=1}^{\infty} E_{j} u$.

(iii) Also, the eigenfunctions $\left\{\varphi_{j k}\right\}_{k=1, j=1}^{m_{j}, \infty} \subset C_{0}^{\infty}(\Omega)$, where $C_{0}^{\infty}(\Omega)$ is the space of infinitely continuously differentiable functions on $\Omega$ and compactly supported in $\Omega$. 
(iv) For all $u \in D(-\Delta)$ we have $-\Delta u=\sum_{j=1}^{\infty} \lambda_{j} E_{j} u$.

(v) The operator $\Delta$ generates an analytic semigroup $\left\{T_{\Delta}(t)\right\}$ on $L^{2}(\Omega)$ defined by

$$
T_{\Delta}(t) u=\sum_{j=1}^{\infty} e^{-\lambda j^{t}} E_{j} u
$$

Definition 2.2. Let $0<\alpha<1$ a real number, the operator $(-\Delta)^{\alpha}$ is defined by

$$
\begin{gathered}
(-\Delta)^{\alpha}: D\left((-\Delta)^{\alpha}\right) \subset L^{2}(\Omega) \longrightarrow L^{2}(\Omega), \\
D\left((-\Delta)^{\alpha}\right)=\left\{\left.u \in L^{2}(\Omega)\left|\sum_{j=1}^{\infty} \sum_{k=1}^{m_{j}}\right| \lambda_{j}^{\alpha}\left\langle u, \varphi_{j k}\right\rangle\right|^{2}<\infty\right\}, \\
(-\Delta)^{\alpha} u=\sum_{j=1}^{\infty} \sum_{k=1}^{m_{j}} \lambda_{j}^{\alpha}\left\langle\varphi_{j k}, u\right\rangle \varphi_{j k} .
\end{gathered}
$$

In particular, we obtain $\varphi_{j k} \in D\left((-\Delta)^{\alpha}\right)$ and $(-\Delta)^{\alpha} \varphi_{j k}=\lambda_{j}^{\alpha} \varphi_{j k}$. Since $\left\{\varphi_{j k}\right\}_{k=1, j=1}^{m_{j}, \infty}$ form a complete orthonormal system in the space $L^{2}(\Omega)$, then it is dense in $L^{2}(\Omega)$, and hence $D(-\Delta)^{\alpha}$ is dense in $L^{2}(\Omega)$.

Proposition 2.3 (cf. [4]). Let $X$ be a Hilbert separable space and $\left\{A_{j}\right\}_{j \geq 1}$ and $\left\{P_{j}\right\}_{j \geq 1}$ two families of bounded linear operators in $X$, with $\left\{P_{j}\right\}_{j \geq 1}$ a family of complete orthogonal projections such that $A_{j} P_{j}=P_{j} A_{j}, j \geq 1$.

Define the following family of linear operators $S(t) w=\sum_{j=1}^{\infty} e^{A_{j} t} P_{j} w, w \in X, t \geq 0$. Then

(a) $S(t)$ is a linear and bounded operator if $\left\|e^{A_{j} t}\right\| \leq g(t), j \geq 1$ with $g(t) \geq 0$, continiuous for $t \geq 0$,

(b) under the above condition (a), $\{S(t)\}_{t \geq 0}$ is a strongly continiuous semigroup in the Hilbert space $X$, whose infinitesimal generator $A$ is given by

$$
A w=\sum_{j=1}^{\infty} A_{j} P_{j} w, \quad w \in D(A), \quad D(A)=\left\{w \in X \mid \sum_{j=1}^{\infty}\left\|A_{j} P_{j} w\right\|^{2}<\infty\right\}
$$

Theorem 2.4 (cf. [5]). Suppose $\Omega$ is connected, $f$ is a real function in $\Omega$, and $f=0$ on a nonempty open subset of $\Omega$. Then $f \equiv 0$ in $\Omega$.

\section{Abstract Formulation of the Problem}

In this section we consider the following notations.

(i) $X=L^{2}(\Omega) \times L^{2}(\Omega)$. X is a Hilbert space with the inner product

$$
\left\langle\left(u_{1}, v_{1}\right),\left(u_{2}, v_{2}\right)\right\rangle=\left\langle u_{1}, u_{2}\right\rangle+\left\langle v_{1}, v_{2}\right\rangle \text {. }
$$


(ii) We define

$$
\begin{aligned}
& A_{11}(u, v)=a_{1} \Delta u+a_{2} \Delta v-c_{1}(-\Delta)^{\alpha_{1}} u-c_{2}(-\Delta)^{\alpha_{2}} v \\
& A_{12}(u, v)=b_{1} \Delta u+b_{2} \Delta v-d_{1}(-\Delta)^{\beta_{1}} u-d_{2}(-\Delta)^{\beta_{2}} v
\end{aligned}
$$

(iii) Let $w=(u, v)$, then we can define the linear operator

$$
\begin{gathered}
A: D(A) \subset X \longrightarrow X, \\
D(A)=\left(H^{2}(\Omega ; \mathbb{R}) \cap H_{0}^{1}(\Omega ; \mathbb{R})\right)^{2}, \\
A w=-\left(M \Delta-c_{1} B_{1}(-\Delta)^{\alpha_{1}}-c_{2} B_{2}(-\Delta)^{\alpha_{2}}-d_{1} B_{3}(-\Delta)^{\beta_{1}}-d_{2} B_{4}(-\Delta)^{\beta_{2}}\right) w,
\end{gathered}
$$

where

$$
\begin{array}{ll}
M=\left(\begin{array}{ll}
a_{1} & a_{2} \\
b_{1} & b_{2}
\end{array}\right), & B_{1}=\left(\begin{array}{ll}
1 & 0 \\
0 & 0
\end{array}\right), \quad B_{2}=\left(\begin{array}{ll}
0 & 1 \\
0 & 0
\end{array}\right), \\
B_{3}=\left(\begin{array}{ll}
0 & 0 \\
1 & 0
\end{array}\right), & B_{4}=\left(\begin{array}{ll}
0 & 0 \\
0 & 1
\end{array}\right) .
\end{array}
$$

Therefore, for all $w \in D(A)$

$$
\begin{aligned}
& A_{11}(u, v)=a_{1} \sum_{j=1}^{\infty} \lambda_{j} E_{j} u+a_{2} \sum_{j=1}^{\infty} \lambda_{j} E_{j} v+c_{1} \sum_{j=1}^{\infty} \lambda_{j}^{\alpha_{1}} E_{j} u+c_{2} \sum_{j=1}^{\infty} \lambda_{j}^{\alpha_{2}} E_{j} v, \\
& A_{12}(u, v)=b_{1} \sum_{j=1}^{\infty} \lambda_{j} E_{j} u+b_{2} \sum_{j=1}^{\infty} \lambda_{j} E_{j} v+d_{1} \sum_{j=1}^{\infty} \lambda_{j}^{\beta_{1}} E_{j} u+d_{2} \sum_{j=1}^{\infty} \lambda_{j}^{\beta_{2}} E_{j} v .
\end{aligned}
$$

If we put

$$
P_{j}=\left(\begin{array}{cc}
E_{j} & 0 \\
0 & E_{j}
\end{array}\right), \quad j=1,2
$$

then (3.3) can be written as

$$
A w \equiv\left(\begin{array}{l}
A_{11}(u, v) \\
A_{12}(u, v)
\end{array}\right)=\sum_{j=1}^{\infty}\left(\lambda_{j} M+\lambda_{j}^{\alpha_{1}} c_{1} B_{1}+\lambda_{j}^{\alpha_{2}} c_{2} B_{2}+\lambda_{j}^{\beta_{1}} d_{1} B_{3}+\lambda_{j}^{\beta_{2}} d_{2} B_{4}\right) P_{j} w,
$$

and we have for all $w \in X$

$$
w=\sum_{j=1}^{\infty} P_{j} w, \quad\|w\|^{2}=\sum_{j=1}^{\infty}\left\|P_{j} w\right\|^{2}
$$


Consequently, system (1.1) can be written as an abstract differential equation in the Hilbert space $X$ in the following form:

$$
\begin{gathered}
\left.\dot{w}=-A w+B_{\omega} f(t), \quad \text { in } \Omega \times\right] 0, t^{*}[, \\
w=0, \quad \text { on }] 0, t^{*}[\times \partial \Omega, \\
w(0)=w_{0}, \quad \text { in } x \in \Omega,
\end{gathered}
$$

where $\left.f \equiv \operatorname{col}\left(f_{1}, f_{2}\right) \in L^{2}([0, T] ; X)\right)$ and $B_{\omega}=\left(\begin{array}{cc}1_{\omega} & 0 \\ 0 & 1_{\omega}\end{array}\right)$ is a bounded linear operator from $U$ into $X$.

\section{Main Results}

\subsection{Generation of a $C_{0}$-Semigroup}

Theorem 4.1. If $c_{2}=d_{1}=0$, then, under hypotheses (H1)-(H3), the linear operator $-A$ defined by (3.3) is the infinitesimal generator of strongly continuous semigroup $\{S(t)\}_{t \geq 0}$ given by

$$
S(t) w=\sum_{j=1}^{\infty} e^{A_{j} t} P_{j} w, \quad w \in X
$$

where

$$
\begin{gathered}
M_{j}=-\lambda_{j} M-\lambda_{j}^{\alpha_{1}} c_{1} B_{1}-\lambda_{j}^{\alpha_{2}} c_{2} B_{2}-\lambda_{j}^{\beta_{1}} d_{1} B_{3}-\lambda_{j}^{\beta_{2}} d_{2} B_{4}, \\
A_{j}=M_{j} P_{j} .
\end{gathered}
$$

Moreover, if

$$
\min \left\{c_{1}+\lambda_{1}^{1-\alpha_{1}} \rho_{1}, d_{2}+\lambda_{1}^{1-\beta_{2}} \rho_{1}\right\}>0
$$

then the $C_{0}$-semigoup $\{S(t)\}_{t \geq 0}$ is exponentially stable, that is, there exist two positives constants $c, \delta$ such that

$$
\|S(t)\| \leq c e^{-\delta t}, \text { for all } t \geq 0
$$

Proof. In order to apply the Proposition 2.3, we observe that $-A$ can be written as follows:

$$
-A w=\sum_{j=1}^{\infty} A_{j} P_{j} w, \quad w \in D(A)
$$


where

$$
A_{j}=-\left(\lambda_{j} M+\lambda_{j}^{\alpha_{1}} c_{1} B_{1}+\lambda_{j}^{\alpha_{2}} c_{2} B_{2}+\lambda_{j}^{\beta_{1}} d_{1} B_{3}+\lambda_{j}^{\beta_{2}} d_{2} B_{4}\right) P_{j} .
$$

Therefore, $A_{j}=M_{j} P_{j}$ and $A_{j} P_{j}=P_{j} A_{j}$.

Now, we have to verify condition (a) of the Proposition 2.3. We shall suppose that $0<\rho_{1}<\rho_{2}$. Then, there exists a set $\left\{Q_{1}, Q_{2}\right\} \in\left[\mathcal{M}_{2}(\mathbb{R})\right]^{2}$ of complementary projections on $\mathbb{R}^{2}$ such that

$$
e^{M t}=e^{\rho_{1} t} Q_{1}+e^{\rho_{2} t} Q_{2} .
$$

If $G=\left(\begin{array}{ll}g_{11} & g_{12} \\ g_{21} & g_{22}\end{array}\right)$ is the matrix passage from the canonical basis of $\mathbb{R}^{2}$ to the basis composed with the eigenvectors of $M$, then

$$
Q_{1}=\frac{1}{\rho_{1} \rho_{2}}\left(\begin{array}{ll}
g_{11} g_{22} & -g_{11} g_{12} \\
g_{21} g_{22} & -g_{12} g_{21}
\end{array}\right), \quad Q_{2}=\frac{1}{\rho_{1} \rho_{2}}\left(\begin{array}{ll}
-g_{12} g_{21} & g_{11} g_{12} \\
-g_{21} g_{22} & g_{11} g_{22}
\end{array}\right) .
$$

Hence,

$$
e^{-\lambda_{j} M t}=e^{-\lambda_{j} \rho_{1} t} Q_{1}+e^{-\lambda_{j} \rho_{2} t} Q_{2} .
$$

We have also

$$
\begin{array}{ll}
e^{-\lambda_{j}^{\alpha_{1}} c_{1} B_{1} t}=\left(\begin{array}{cc}
e^{-\lambda_{j}^{\alpha_{1}} c_{1} t} & 0 \\
0 & 1
\end{array}\right), & e^{-\lambda_{j}^{\alpha_{2}} c_{2} B_{2} t}=\left(\begin{array}{cc}
1 & -\lambda_{j}^{\alpha_{2}} c_{2} t \\
0 & 1
\end{array}\right), \\
e^{-\lambda_{j}^{\beta_{1}} d_{1} B_{3} t}=\left(\begin{array}{cc}
1 & 0 \\
-\lambda_{j}^{\beta_{1}} d_{1} t & 1
\end{array}\right), & e^{-\lambda_{j}^{\beta_{1}} d_{2} B_{4} t}=\left(\begin{array}{cc}
1 & 0 \\
0 & e^{-\lambda_{j}^{\beta_{2}} d_{2} t}
\end{array}\right) .
\end{array}
$$

From (4.10)-(4.11) into (4.7) we obtain

$$
e^{A_{j} t}=\left(e^{-\lambda_{j} \rho_{1} t} Q_{1}+e^{-\lambda_{j} \rho_{2} t} Q_{2}\right) K_{j}(t) P_{j}
$$

where

$$
K_{j}(t)=\left(\begin{array}{cc}
e^{-\lambda_{j}^{\alpha_{1}} c_{1} t}+\lambda_{j}^{\alpha_{2}+\beta_{1}} c_{2} d_{1} t^{2} e^{-\lambda_{j}^{\alpha_{1}} c_{1} t} & -\lambda_{j}^{\alpha_{2}} c_{2} t e^{-\left(\lambda_{j}^{\alpha_{1}} c_{1}+\lambda_{j}^{\beta_{2}} d_{2}\right) t} \\
-\lambda_{j}^{\beta_{1}} d_{1} t & e^{-\lambda_{j}^{\beta_{2}} d_{2} t}
\end{array}\right)
$$


As $c_{2}=d_{1}=0$ we get

$$
K_{j}(t)=\left(\begin{array}{cc}
e^{-\lambda_{j}^{\alpha_{1}} c_{1} t} & 0 \\
0 & e^{-\lambda_{j}^{\beta_{2}} d_{2} t}
\end{array}\right) .
$$

As $\lambda_{j} \rightarrow+\infty$ as $j \rightarrow \infty$, then this implies the existence of a positive number $c$ and a real number $\delta$ such that $\left\|e^{A_{j} t}\right\| \leq c e^{\delta t}$, for every $j \geq 1$. Therefore $-A$ is a strongly continious semigroup $\{S(t)\}_{t \geq 0}$ given by (4.1). We can even estimate the constants $c$ and $\delta$ as follows.

(i) If $\min \left\{c_{1}+\lambda_{1}^{1-\alpha_{1}} \rho_{1}, d_{2}+\lambda_{1}^{1-\beta_{2}} \rho_{1}\right\} \leq 0$. As $\lim _{j \rightarrow \infty}\left\{-\lambda_{j}^{\alpha_{1}}\left(c_{1}+\lambda_{j}^{1-\alpha_{1}} \rho_{1}\right)\right\}=$ $\lim _{j \rightarrow \infty}\left\{-\lambda_{j}^{\beta_{2}}\left(c_{1}+\lambda_{j}^{1-\beta_{2}} \rho_{1}\right)\right\}=-\infty$, then there exist constants

$$
\begin{aligned}
& \delta_{1}=\max \left\{-\lambda_{j}^{\alpha_{1}}\left(c_{1}+\lambda_{j}^{1-\alpha_{1}} \rho_{1}\right) \mid \lambda_{j}^{\alpha_{1}}\left(c_{1}+\lambda_{j}^{1-\alpha_{1}} \rho_{1}\right) \leq 0, j \geq 1\right\}, \\
& \delta_{2}=\max \left\{-\lambda_{j}^{\beta_{2}}\left(d_{2}+\lambda_{j}^{1-\beta_{2}} \rho_{1}\right) \mid \lambda_{j}^{\beta_{2}}\left(d_{2}+\lambda_{j}^{1-\beta_{2}} \rho_{1}\right) \leq 0, j \geq 1\right\},
\end{aligned}
$$

hence, if we put

$$
\begin{gathered}
\delta=\max \left\{\delta_{1}, \delta_{2}\right\} \geq 0, \\
c_{0}=\frac{1}{\rho_{1} \rho_{2}} \max \left\{\left|g_{11} g_{22}\right|,\left|g_{11} g_{12}\right|,\left|g_{21} g_{22}\right|,\left|g_{12} g_{21}\right|\right\},
\end{gathered}
$$

we easily obtain

$$
\left\|e^{A_{j} t}\right\| \leq 4 c_{0} e^{-\delta t}, \quad j \geq 1 .
$$

(ii) If $\min \left\{c_{1}+\lambda_{1}^{1-\alpha_{1}} \rho_{1}, d_{2}+\lambda_{1}^{1-\beta_{2}} \rho_{1}\right\}>0$. If we put

$$
\delta=\min \left\{\lambda_{1}^{\alpha 1}\left(c_{1}+\lambda_{1}^{1-\alpha 1} \rho_{1}\right), \lambda_{1}^{\beta_{2}}\left(d_{2}+\lambda_{1}^{1-\beta_{2}} \rho_{1}\right)\right\}>0,
$$

then we find that

$$
\left\|e^{A_{j} t}\right\| \leq 4 c_{0} e^{-\delta t}, \quad j \geq 1 .
$$

Therefore, the linear operator $-A$ generates a strongly continuous semigroup $\{S(t)\}_{t \geq 0}$ on $X$ given by expression (4.1).

Finally, if $\min \left\{c_{1}+\lambda_{1}^{1-\alpha_{1}} \rho_{1}, d_{2}+\lambda_{1}^{1-\beta_{2}} \rho_{1}\right\}>0$, we have already proved (4.20). Using (4.20) into (4.1) we get that the $C_{0}$-semigoup $\{S(t)\}_{t \geq 0}$ is exponentially stable. The expression (4.5) is verfied with $c=4 c_{0}$ and $\delta$ is defined by (4.19). 
Theorem 4.2. If

$$
c_{2} \neq 0, \quad d_{1} \neq 0, \quad c_{1} \geq 0, \quad d_{2} \geq 0,
$$

then, under the hypotheses (H1)-(H3), the linear operator - A defined by (3.3) is the infinitesimal generator of strongly continuous semigroup exponentially stable $\{S(t)\}_{t \geq 0}$ defined by (4.1). Specially, there exist two positives constants $c, \delta$ such that

$$
\|S(t)\| \leq c e^{-\delta t}, \quad \forall t \geq 0
$$

To prove this result, we need the following lemma.

Lemma 4.3. For every two real positives constants $c$ and $\lambda$, one has for every $0<\delta<\lambda / c$

$$
c t e^{-\lambda t} \leq \frac{1}{e(\lambda / c-\delta)} e^{-\delta c t}, \quad \forall t \geq 0,
$$

and for every $0<\delta<\lambda / \sqrt{c}$

$$
c t^{2} e^{-\lambda t} \leq \frac{4}{e^{2}(\lambda / \sqrt{c}-\delta)} e^{-\delta \sqrt{c} t}, \quad \forall t \geq 0
$$

Proof of Lemma 4.3. It is easy to verify that for every $\varepsilon>0: t e^{-\varepsilon t} \leq 1 / e \varepsilon$, for all $t \geq 0$.

Let $0<\delta<\lambda / c$ and $\varepsilon=\lambda / c-\delta>0$, then we get

$$
t e^{(-\lambda / c t)} \leq \frac{1}{e(\lambda / c-\delta)} e^{-\delta t}, \quad \forall t \geq 0
$$

Hence, we get (4.23).

Also, it is easy to verify that for every $\varepsilon>0: t^{2} e^{-\varepsilon t} \leq 4 / e^{2} \varepsilon^{2}$, for all $t \geq 0$. Let $0<\delta<\lambda / \sqrt{c}$ and $\varepsilon=\lambda / \sqrt{c}-\delta>0$, then we get

$$
t^{2} e^{-(\lambda \sqrt{c}) t} \leq \frac{4}{e^{2}((\lambda / \sqrt{c})-\delta)^{2}} e^{-\delta t}, \quad \forall t \geq 0
$$

Hence, from (4.26) we get $c t^{2} e^{-\lambda t}=(\sqrt{c} t)^{2} e^{-(\lambda / \sqrt{c}) \sqrt{c} t} \leq 4 / e^{2}(\lambda / \sqrt{c}-\delta)^{2} e^{-\delta \sqrt{c} t}$ for all $t \geq 0$ and $0<\delta<\lambda / \sqrt{c}$, which gives (4.24). we have

With the same manner we can prove that for every $0<\delta<\lambda c^{-1 / n}$ and every $n \in \mathbb{N}^{*}$

$$
t^{n} e^{-\lambda c^{-1 / n} t} \leq \frac{n^{n}}{(e \varepsilon)^{n}} e^{-\delta t}, \quad \forall t \geq 0
$$


and consequently, for every two real positives constants $c$ and $\lambda$ and every $n \in \mathbb{N}^{*}$ we have

$$
c t^{n} e^{-\lambda t} \leq \frac{n^{n}}{(e \varepsilon)^{n}} e^{-\delta c^{-1 / n} t}, \quad \text { for all } t \geq 0 \text { and every } 0<\delta<\lambda .
$$

Now, we are ready to prove Theorem 4.2.

Proof of Theorem 4.2. By applying Proposition 2.3 we start from formula (4.12) and we put

$$
K_{j}(t)=\left(\begin{array}{ll}
K_{11, j}(t) & K_{12, j}(t) \\
K_{21, j}(t) & K_{22, j}(t)
\end{array}\right)
$$

where

$$
\begin{aligned}
& K_{11, j}(t)=e^{-\lambda_{j}^{\alpha_{1}} c_{1} t}+\lambda_{j}^{\alpha_{2}+\beta_{1}} c_{2} d_{1} t^{2} e^{-\lambda_{j}^{\alpha_{1}} c_{1} t}, \quad K_{12, j}(t)=-\lambda_{j}^{\alpha_{2}} c_{2} t e^{-\left(\lambda_{j}^{\alpha_{1}} c_{1}+\lambda_{j}^{\beta_{2}} d_{2}\right) t}, \\
& K_{21, j}(t)=-\lambda_{j}^{\beta_{1}} d_{1} t, \quad K_{22, j}(t)=e^{-\lambda_{j}^{\beta_{2}} d_{2} t}, \quad \forall j \geq 1 .
\end{aligned}
$$

To estimate $e^{-\lambda_{j} \rho_{1} t} K_{11, j}(t)$ we have in taking into account $c_{1} \geq 0$

$$
e^{-\left(\lambda_{j} \rho_{1}+\lambda_{j}^{\alpha_{1}} c_{1}\right) t} \leq e^{-\lambda_{1} \rho_{1} t}, \quad \forall t \geq 0,
$$

and applying the Lemma $4.3\left(c=\lambda_{j}^{\alpha_{2}+\beta_{1}}\left|c_{2} d_{1}\right|\right)$ we get

$$
\begin{aligned}
& \lambda_{j}^{\alpha_{2}+\beta_{1}} c_{2} d_{1} t^{2} e^{-\left(\lambda_{j} \rho_{1}+\lambda_{j}^{\alpha_{1}} c_{1}\right) t} \\
& \quad \leq \frac{4}{e^{2}\left(\left(\lambda_{j}^{1-\left(\alpha_{2}+\beta_{1}\right) / 2} / \sqrt{\left|c_{2} d_{1}\right|}\right) \rho_{1}+\left(\lambda_{j}^{\alpha_{1}-\left(\alpha_{2}+\beta_{1} / 2\right)} / \sqrt{\left|c_{2} d_{1}\right|}\right) c_{1}-\gamma_{1}\right)} e^{-\gamma_{1} \lambda_{j}^{\alpha_{2}+\beta_{1} / 2} \sqrt{\left|c_{2} d_{1}\right|}},
\end{aligned}
$$

for all $t \geq 0$ and $0<\gamma_{1}<\left(\lambda_{j}^{1-\left(\alpha_{2}+\beta_{1}\right) / 2} / \sqrt{\left|c_{2} d_{1}\right|}\right) \rho_{1}+\left(\lambda_{j}^{\alpha_{1}-\left(\alpha_{2}+\beta_{1}\right) / 2} / \sqrt{\left|c_{2} d_{1}\right|}\right) c_{1}$. But we have $\left(\lambda_{j}^{1-\left(\alpha_{2}+\beta_{1}\right) / 2} / \sqrt{\left|c_{2} d_{1}\right|}\right) \rho_{1}+\left(\lambda_{j}^{\alpha_{1}-\left(\alpha_{2}+\beta_{1}\right) / 2} / \sqrt{\left|c_{2} d_{1}\right|}\right) c_{1} \geq\left(\lambda_{1}^{1-\left(\alpha_{2}+\beta_{1}\right) / 2} / \sqrt{\left|c_{2} d_{1}\right|}\right) \rho_{1}$, for all $j \geq 1$. Then we get for every $0<\gamma_{1}<\left(\lambda_{1}^{1-\left(\alpha_{2}+\beta_{1}\right) / 2} / \sqrt{\left|c_{2} d_{1}\right|}\right) \rho_{1}$ that

$$
\begin{aligned}
& \lambda_{j}^{\alpha_{2}+\beta_{1}} c_{2} d_{1} t^{2} e^{-\left(\lambda_{j} \rho_{1}+\lambda_{j}^{\alpha_{1}} c_{1}\right) t} \\
& \quad \leq \frac{4}{e^{2}\left(\left(\lambda_{1}^{1-\left(\alpha_{2}+\beta_{1}\right) / 2} / \sqrt{\left|c_{2} d_{1}\right|}\right) \rho_{1}-\delta_{1}\right)} e^{-\gamma_{1}\left(\lambda_{1}^{\left(\alpha_{2}+\beta_{1}\right) / 2} \sqrt{\left.\left|c_{2} d_{1}\right|\right) t}, \quad \forall t \geq 0 .\right.}
\end{aligned}
$$

From (4.31)-(4.33) we get

$$
e^{-\lambda_{j} \rho_{1} t} K_{11, j}(t) \leq \sigma_{1} e^{-\delta_{1} t}, \quad \forall t \geq 0, j \geq 1,
$$


where

$$
\sigma_{1}=1+4\left(\frac{\lambda_{1}^{1-\left(\alpha_{2}+\beta_{1}\right) / 2}}{\sqrt{\left|c_{2} d_{1}\right|}} \rho_{1}-\delta_{1}\right)^{-1}, \quad \delta_{1}=\min \left\{\lambda_{1} \rho_{1}, \gamma_{1} \lambda_{1}^{\left(\alpha_{2}+\beta_{1}\right) / 2} \sqrt{\left|c_{2} d_{1}\right|}\right\}
$$

and $0<\gamma_{1}<\left(\lambda_{1}^{1-\left(\alpha_{2}+\beta_{1}\right) / 2} / \sqrt{\left|c_{2} d_{1}\right|}\right) \rho_{1}$.

Applying Lemma 4.3 and taking into account (4.21) we get with the same manner that for every $0<\delta_{2}<\left(\lambda_{1}^{1-\alpha_{2}} /\left|c_{2}\right|\right) \rho_{1}$

$$
e^{-\lambda_{j} \rho_{1} t} K_{12, j}(t) \leq \sigma_{2} e^{-\delta_{2} \lambda_{1}^{\alpha_{2}}\left|c_{2}\right| t}, \quad \forall t \geq 0, j \geq 1,
$$

where

$$
\sigma_{2}=\frac{1}{e\left(\left(\lambda_{1}^{1-\alpha_{2}} /\left|c_{2}\right|\right) \rho_{1}-\delta_{2}\right)}
$$

and or every $0<\delta_{3}<\left(\lambda_{1}^{1-\beta_{1}} /\left|d_{1}\right|\right) \rho_{1}$

$$
e^{-\lambda_{j} \rho_{1} t} K_{21, j}(t) \leq \sigma_{3} e^{-\delta_{3} \lambda_{1}^{\beta_{1}}\left|d_{1}\right| t}, \quad \forall t \geq 0, j \geq 1,
$$

where

$$
\begin{gathered}
\sigma_{3}=\frac{1}{e\left(\left(\lambda_{1}^{1-\beta_{1}} /\left|d_{1}\right|\right) \rho_{1}-\delta_{3}\right)}, \\
e^{-\lambda_{j} \rho_{1} t} K_{22, j}(t) \leq e^{-\lambda_{1} \rho_{1} t}, \quad \forall t \geq 0, j \geq 1 .
\end{gathered}
$$

From (4.34)-(4.40) into (4.12) we get

$$
\left\|e^{A_{j} t}\right\| \leq 4 c_{0} \sigma e^{-\delta t}, \quad \forall t \geq 0, j \geq 1
$$

where $c_{0}$ is defined by (4.17) and

$$
\sigma=1+\sigma_{1}+\sigma_{2}+\sigma_{3}, \quad 0<\delta<\min \left\{\delta_{1}, \delta_{2} \lambda_{1}^{\alpha_{2}}\left|c_{2}\right|, \delta_{3} \lambda_{1}^{\beta_{1}}\left|d_{1}\right|, \lambda_{1} \rho_{1}\right\}
$$

Using (4.41) into (4.1) we get that the $C_{0}$-semigoup $\{S(t)\}_{t \geq 0}$ generated by $-A$ is exponentially stable. Expression (4.22) is verfied with $c=4 c_{0} \sigma$ and $\delta$ is defined by (4.42). 


\subsection{Approximate Controllability}

Befor giving the definition of the approximate controllabiliy for the sytem (3.9), we have the following known result: for all $w_{0} \in X$ and $f \in L^{2}(] 0, T[; U)$, the initial value problem (3.9) admits a unique mild solution given by

$$
w(t)=S(t) w_{0}+\int_{0}^{t} S(t-\tau) B_{\omega} f(\tau) d \tau, \quad t \in[0, T]
$$

This solution is denoted by $w(t ; f)$.

Definition 4.4. System (3.9) is said to be approximately controllable at time $t^{*}$ whenever the set $F_{t^{*}}=\left\{w\left(t^{*} ; f\right) \mid \forall f \in L^{2}(] 0, t^{*}[; U)\right\}$ is densely embedded in $X$; that is,

$$
\forall w_{0}, w_{1} \in X, \forall \varepsilon>0 ; \exists f \in L^{2}(] 0, t^{*}[; U):\left\|w\left(t^{*} ; f\right)-w_{1}\right\|<\varepsilon
$$

The following criteria for approximate controllability can be found in [6].

Criteria 1. System (3.9) is approximately controllable on $\left[0, t^{*}\right]$ if and only if

$$
B^{*} S^{*}(t) w=0, \quad \forall t \in\left[0, t^{*}\right] \Longrightarrow w=0
$$

Now, we are ready to formulate the third main result of this work.

Theorem 4.5. If the following condition

$$
c_{2}=d_{1}=0
$$

is satisfied; then, under hypotheses (H1)-(H5), for all $t^{*}>0$ and all open subset $\omega \subset \Omega$, system (3.9) is approximately controllable on $\left[0, t^{*}\right]$.

Proof. The proof of this theorem relies on the Criteria 1 and the following lemma.

Lemma 4.6. Let $\left\{\alpha_{1 j}\right\}_{j \geq 1},\left\{\beta_{1 j}\right\}_{j \geq 1}$ and $\left\{\alpha_{2 j}\right\}_{j \geq 1},\left\{\beta_{2 j}\right\}_{j \geq 1}$ be sequences of real numbers such that $\alpha_{11}>\alpha_{12}>\alpha_{13}>\cdots, \alpha_{21}>\alpha_{22}>\alpha_{23}>\cdots$ and $\alpha_{1 j}>\alpha_{2 j}$, for all $j \geq 0$, then for any $t^{*} \in \mathbb{R}_{+}^{*}$ one has

$$
\sum_{j=1}^{\infty}\left(e^{\alpha_{1 j} t} \beta_{1 j}+e^{\alpha_{2 j} t} \beta_{2 j}\right)=0, \quad \forall t \in\left[0, t^{*}\right] \Longrightarrow \beta_{1 j}=\beta_{2 j}=0, \forall j \geq 1
$$

Proof of Lemma 4.6. By analyticity we get $\sum_{j=1}^{\infty}\left(e^{\alpha_{1 j} t} \beta_{1 j}+e^{\alpha_{2 j} t} \beta_{2 j}\right)=0, \forall t \geq 0$ and from this we get $\beta_{11}+\sum_{j=2}^{\infty} e^{\left(\alpha_{1 j}-\alpha_{11}\right) t} \beta_{1 j}+\sum_{j=1}^{\infty} e^{\left(\alpha_{2 j}-\alpha_{11}\right) t} \beta_{2 j}=0, \forall t \geq 0$. Under the assumptions of the lemma we get $\sum_{j=2}^{\infty} e^{\left(\alpha_{1 j}-\alpha_{11}\right) t} \beta_{1 j}+\sum_{j=1}^{\infty} e^{\left(\alpha_{2 j}-\alpha_{11}\right) t} \beta_{2 j} \rightarrow 0$ as $t \rightarrow \infty$ and so $\beta_{11}=0$. If $\alpha_{12}>\alpha_{21}$, we divide $\sum_{j=2}^{\infty} e^{\alpha_{1 j} t} \beta_{1 j}+\sum_{j=1}^{\infty} e^{\alpha_{2 j} t} \beta_{2 j}=0$ by $e^{\alpha_{12} t}$ and we pass $t \rightarrow \infty$ we get $\beta_{12}=0$. If $\alpha_{21}>\alpha_{12}$, we divide $\sum_{j=2}^{\infty} e^{\alpha_{1 j} t} \beta_{1 j}+\sum_{j=1}^{\infty} e^{\alpha_{2 j} t} \beta_{2 j}=0$ by $e^{\alpha_{21} t}$ and we pass $t \rightarrow \infty$ and get $\beta_{21}=0$. If $\alpha_{12}=\alpha_{21}$, 
we divide $\sum_{j=2}^{\infty} e^{\alpha_{1 j} t} \beta_{1 j}+\sum_{j=1}^{\infty} e^{\alpha_{2 j} t} \beta_{2 j}=0$ by $e^{\alpha_{12} t}$ and we pass $t \rightarrow \infty$ and get $\beta_{12}+\beta_{21}=0$. But in this we case we can integrate under the symbol of sommation over the intervall $[0, t]$ and we get $\beta_{12} e^{\alpha_{21} t}+\beta_{21} e^{\alpha_{12} t}=0$. Hence $\beta_{12}=\beta_{21}=0$. Continuing this way we see that $\beta_{1 j}=\beta_{2 j}=0$, for all $j \geq 1$.

We are now ready to prove Theorem 4.5. For this purpose, we observe that

$$
B_{\omega}^{*}=B_{\omega}, \quad S^{*}(t) w=\sum_{j=1}^{\infty} e^{M_{j}^{*} t} P_{j}^{*} w, \quad w \in X, t \geq 0
$$

where $\{S(t)\}_{t \geq 0}$ is the $C_{0}$-semigroup generated by $-A$.

Without lose of generality, we suppose that $0<\rho_{1}<\rho_{2}$. Hence

$$
B_{\omega}^{*} S^{*}(t) w=\sum_{j=1}^{\infty} B_{\omega}^{*} e^{M_{j}^{*} t} P_{j}^{*} w=\sum_{j=1}^{\infty} B_{\omega}^{*} e^{M_{j}^{*} t} P_{j}^{*} w=\sum_{j=1}^{\infty} \sum_{s=1}^{2} B_{\omega}^{*} K_{j}^{*}(t)\left(e^{-\lambda_{j} \rho_{s} t} P_{s j}^{*}\right) w,
$$

where $P_{s j}=Q_{s} P_{j}=P_{j} Q_{s}, s=1,2$.

Now, suppose for $w \in X$ that $B_{\omega}^{*} S^{*}(t) w=0$, for all $t \in\left[0, t^{*}\right]$. Then

$$
B_{\omega}^{*} S^{*}(t) w=0 \Longleftrightarrow \sum_{j=1}^{\infty} \sum_{s=1}^{2} B_{\omega}^{*} K_{j}^{*}(t)\left(e^{-\lambda_{j} \rho_{s} t} P_{s j}^{*}\right) w(x)=0, \quad \forall x \in \Omega .
$$

If (4.46) is satisfied, then (4.50) take the form

$$
\sum_{j=1}^{\infty} \sum_{s=1}^{2}\left(\begin{array}{cc}
e^{-\left(\lambda_{j} \rho_{s}+\lambda_{j}^{\alpha_{1}} c_{1}\right) t} & 0 \\
0 & e^{-\left(\lambda_{j} \rho_{s}+\lambda_{j}^{\beta_{2}} d_{2}\right) t}
\end{array}\right)\left(B_{\omega}^{*} P_{s j}^{*}\right) w(x)=0, \quad \forall x \in \Omega .
$$

Then, from lemma 4.6 we obtain that for $s=1,2$ and all $x \in \omega$

$$
\left(B_{\omega}^{*} Q_{s}^{*} P_{j}^{*} w\right)(x)=Q_{s}^{*}\left(\begin{array}{l}
\sum_{k=1}^{m_{j}}\left\langle u, \varphi_{j k}\right\rangle 1_{\omega} \varphi_{j k}(x) \\
\sum_{k=1}^{m_{j}}\left\langle v, \varphi_{j k}\right\rangle 1_{\omega} \varphi_{j k}(x)
\end{array}\right)=\left(\begin{array}{l}
0 \\
0
\end{array}\right), \quad j \geq 1 .
$$

Since $Q_{1}+Q_{2}=I_{\mathbb{R}^{2}}$, we get that all $x \in \omega$

$$
\left(\begin{array}{l}
\sum_{k=1}^{m_{j}}\left\langle u, \varphi_{j k}\right\rangle 1_{\omega} \varphi_{j k}(x) \\
\sum_{k=1}^{m_{j}}\left\langle v, \varphi_{j k}\right\rangle 1_{\omega} \varphi_{j k}(x)
\end{array}\right)=\left(\begin{array}{l}
0 \\
0
\end{array}\right), \quad s=1,2, j \geq 1 .
$$


On the other hand, from Theorem 2.4 we know that $\varphi_{j k}$ are analytic functions, which implies the analticity of $E_{j} u=\sum_{k=1}^{m_{j}}\left\langle u, \varphi_{j k}\right\rangle \varphi_{j k}$ and $E_{j} v=\sum_{k=1}^{m_{j}}\left\langle v, \varphi_{j k}\right\rangle \varphi_{j k}$. Then we can conclude that for $s=1,2$ and all $x \in \Omega$

$$
\left(\begin{array}{l}
\sum_{k=1}^{m_{j}}\left\langle u, \varphi_{j k}\right\rangle \varphi_{j k}(x) \\
\sum_{k=1}^{m_{j}}\left\langle v, \varphi_{j k}\right\rangle \varphi_{j k}(x)
\end{array}\right)=\left(\begin{array}{l}
0 \\
0
\end{array}\right), \quad j \geq 1 .
$$

Hence $P_{j} w=0$, for all $j \geq 1$, which implies that $w=0$. This completes the proof of Theorem 4.5.

\section{References}

[1] S. Badraoui, "Asymptotic behavior of solutions to a $2 \times 2$ reaction-diffusion system with a cross diffusion matrix on unbounded domains," Electronic Journal of Differential Equations, vol. 2006, no. 61, pp. 1-13, 2006.

[2] H. Larez and H. Leiva, "Interior controllability of a $2 \times 2$ a reaction-diffusion system with cross diffusion matrix," to appear in Boundary Value Problems.

[3] E. Zeidler, Applied Functional Analysis, vol. 109 of Applied Mathematical Sciences, Springer, New York, NY, USA, 1995.

[4] H. Leiva, "A lemma on $\mathrm{C}_{0}$-semigroups and applications," Quaestiones Mathematicae, vol. 26, no. 3, pp. 247-265, 2003.

[5] S. Axler, P. Bourdon, and W. Ramey, Harmonic Function Theory, vol. 137 of Graduate Texts in Mathematics, Springer, New York, NY, USA, 1992.

[6] R. F. Curtain and H. Zwart, An Introduction to Infinite-Dimensional Linear Systems Theory, vol. 21 of Texts in Applied Mathematics, Springer, New York, NY, USA, 1995. 